\title{
Prevalência de lesões intraepiteliais em atipias de significado indeterminado em um serviço público de referência para neoplasias cervicais*
}

\author{
Prevalence of atypical squamous cell intraepithelial lesions of undetermined significance in a public \\ bealth referral service for cervical cancer
}

Prevalencia de lesiones intraepiteliales en atipias de significado indeterminado en un servicio público de referencia para neoplasias cervicales

\section{Railda Fraga Costaํ, Sonia Maria Oliveira de Barros ${ }^{2}$}

\begin{abstract}
RESUMO
Objetivos: Determinar a prevalência de lesões intraepiteliais de baixo e alto grau, em mulheres com diagnóstico colpocitológico de atipias de significado indeterminado no Município de Maceió, Alagoas. Conhecer a influência da idade e dos agentes etiológicos para doenças sexualmente transmissíveis sobre o risco de desenvolvimento das lesões intraepiteliais de baixo e alto grau. Métodos: Estudo transversal de prevalência em um serviço público de referência para neoplasias cervicais. Foram estudados 253 prontuários de mulheres com diagnóstico de atipias de significado indeterminado, no ano de 2007. Resultados: A prevalência de lesões intraepiteliais foi a de $23,7 \%$, sendo $26,7 \%$ de baixo grau e 73,3\% de alto grau. Conclusões: A prevalência de lesões intraepiteliais de alto grau em mulheres com diagnóstico colpocitológico de atipias de significado indeterminado (ASCUS) foi de 23,7\%, o aumento do risco foi diretamente proporcional ao da idade e houve maior prevalência de lesão intraepitelial de baixo grau,associada à infecção pelo Papilomavirus humano.

Descritores: Enfermagem; Neoplasia intraepitelial cervical; Prevalência; Saúde da Mulher
\end{abstract}

\begin{abstract}
Objective: To determine the prevalence of low and high grade intraepithelial lesions in women with Pap smear diagnosis of atypical squamous cells of undetermined significance, in the city of Maceió, Alagoas. Knowing the influence of age and the etiological agents for sexually transmitted diseases on the risk of developing low and high grade intraepithelial lesions. Methods: Cross sectional prevalence study at a public referral center for cervical cancer. We studied records of 253 women with atypical diagnoses of undetermined significance, in 2007. Results: The prevalence of intraepithelial lesions was $23.7 \% ; 26.7 \%$ were low grade and $73.3 \%$ high grade. Conclusions: The prevalence of high-grade intraepithelial lesions in women with Pap smear diagnosis of atypical squamous cells of undetermined significance (ASCUS) was $23.7 \%$; the increase in risk was directly proportional to the age and higher prevalence of low grade squamous intraepithelial lesion associated with papillomavirus infection.
\end{abstract}

Keywords: Nursing; Cervical intraepithelial neoplasia; Prevalence; Women's health

\section{RESUMEN}

Objetivos: Determinar la prevalencia de lesiones intraepiteliales de bajo y alto grado, en mujeres con diagnóstico colpocitológico de atipias de significado indeterminado en el Municipio de Maceió, Alagoas. Conocer la influencia de la edad y de los agentes etiológicos para enfermedades sexualmente transmisibles sobre el riesgo de desarrollo de las lesiones intraepiteliales de bajo y alto grado. Métodos: Se trata de un estudio transversal de prevalencia realizado en un servicio público de referencia para neoplasias cervicales. Fueron estudiadas 253 historias clínicas de mujeres con diagnóstico de atipias de significado indeterminado, en el año 2007. Resultados: La prevalencia de lesiones intraepiteliales fue del $23,7 \%$, siendo $26,7 \%$ de bajo grado y $73,3 \%$ de alto grado. Conclusiones: La prevalencia de lesiones intraepiteliales de alto grado en mujeres con diagnóstico colpocitológico de atipias de significado indeterminado (ASCUS) fue del 23,7\%, el aumento del riesgo fue directamente proporcional al de la edad y hubo mayor prevalencia de lesión intraepitelial de bajo grado, asociada a la infección por el Papilomavirus humano. Descriptores: Enfermería; Neoplasia intraepithelial del cuello uterino; Prevalencia; Salud de la mujer

* Artigo extraído da tese de Mestrado "Prevalência de lesões intraepiteliais de baixo e alto grau em mulheres com diagnóstico colpocitológico de atipias de significado indeterminado no município de Maceió, Alagoas" apresentada ao Programa de Pós-Graduação em Enfermagem da Universidade Federal de São Paulo - UNIFESP - São Paulo (SP), Brasil.

${ }^{1}$ Mestre em Ciências. Professora Assistente da Faculdade Integrada Tiradentes, Maceió (AL), Brasil.

${ }^{2}$ Livre Docente. Doutora em Enfermagem. Professora Associada da Disciplina de Enfermagem Obstétrica, Universidade Federal de São Paulo - UNIFESP, São Paulo (SP), Brasil. 


\section{INTRODUÇÃO}

O câncer de colo uterino é um importante problema de saúde pública em decorrência da crescente exposição a fatores de risco e modificação de hábitos de vida da população. Ao contrário do que ocorrem em países desenvolvidos, no Brasil as taxas de mortalidade por câncer de colo de útero continuam elevadas, sendo considerado o segundo tipo de câncer mais comum entre as mulheres, realidade esta refletida também em Maceió, capital do Estado de Alagoas, onde a estimativa do número de casos novos por câncer de colo de útero para 2010 foi de 110/ 100.000 casos, correspondendo à taxa bruta de $22,49^{(1)}$.

Como estratégia do Ministério da Saúde brasileiro foram implementadas ações governamentais e individuais, com o objetivo de aumentar a cobertura populacional do exame de Papanicolaou. O Ministério da Saúde, por meio do Instituto Nacional de Câncer, lançou o Programa Viva Mulher em março de 1996. O objetivo do Programa é diminuir a incidência e a mortalidade por câncer de colo do útero, por meio da ampliação do acesso das mulheres ao exame colpocitológico pelo método de $\mathrm{Pa}$ panicolaou que permite a detecção precoce de lesões precursoras de neoplasias do colo uterino e as alterações citopáticas pelo Papilomavírus humano (HPV), priorizando as de maior risco, garantindo diagnóstico, tratamento e seguimento adequados ${ }^{(2)}$.

A população da cidade de Maceió apresenta características de vida precárias, neste município, predominam mulheres de baixo nível socioeconômico e cultural, condições relacionadas ao câncer cervical. Em 2009, Maceió possuía uma população total de 933.313 habitantes, com 495.977 mulheres. Dessas, 357.105 tinham entre 15 e $69 \operatorname{anos}^{(3)}$.

Não se conhece a verdadeira cobertura obtida com a citologia oncótica, a proporção de mulheres com exames colpocitológicos alterados, nem se receberam tratamento nos casos indicados e também se houve acompanhamento. Desta forma, não está claro se os recursos financeiros para o desenvolvimento de ações para o controle do câncer cervical no Município são suficientes e utilizados com eficiência.

Os programas de rastreamento devem priorizar a alta efetividade e o menor custo possível; para tanto, é necessário garantir a organização, a integralidade e a qualidade do programa, bem como o acompanhamento das pacientes.

A colpocitologia oncológica é o método de eleição no rastreamento do câncer de colo de útero. Para uniformizar o sistema de terminologias para este método diagnóstico, foi criado o Sistema Bethesda em uma reunião de especialistas realizada na cidade de mesmo nome em Maryland (EUA), em 1982. Seu objetivo era estabelecer normas de classificação citológica para reduzir confusões diagnósticas entre alterações celulares benignas e realmente atípicas ${ }^{(4)}$.

A reunião de especialistas realizada na cidade de Bethesda em 2001, resultou em nova classificação citológica de ASCUS. Esta categoria foi reclassificada em "ASCUS" - "células escamosas atípicas, de significado indeterminado"- e "ASC-H" - "células escamosas atípicas não se podendo excluir lesão intraepitelial de alto grau". O objetivo era diferenciar os casos em que há maior probabilidade de existir lesão precursora de câncer de colo uterino e que, por esse motivo, devem ser encaminhados imediatamente para a colposcopia.

Embora esta proposta de subclassificação que anteriormente se denominava ASCUS sem qualquer especificação ter sido discutida e adotada pela Sociedade Brasileira de Citopatologia, a partir de 2002, seu uso ainda não foi observado pela maioria dos clínicos. Em consequência, continuam as dúvidas quanto à necessidade de encaminhamento imediato dos casos para colposcopia. Vários trabalhos relacionam esta categoria citológica à presença de lesões intraepiteliais ou carcinoma de colo uterino em porcentagens variáveis ${ }^{(5-6)}$.

O interesse para a realização deste trabalho surgiu em razão de minha experiência profissional como enfermeira assistencial do Programa de Atenção à Saúde da Mulher do Município de Maceió, Alagoas, tendo prestado assistência de enfermagem às mulheres com alterações colpocitológicas, seja na prevenção ou no acompanhamento. A oportunidade de trabalhar em um programa de referência e realizar assistência a estes casos suscitou a necessidade de evidenciar o comportamento de algumas variáveis nesta população, determinando a prevalência de lesões intraepiteliais de baixo e alto grau em mulheres com diagnóstico colpocitológico de atipias escamosas de significado indeterminado visando a colaborar com a qualidade da assistência prestada nessa instituição.

Desse modo, os objetivos do estudo foram: determinar a prevalência de lesões intraepiteliais de baixo e alto grau, em mulheres com diagnóstico colpocitológico de atipias de significado indeterminado no Município de Maceió, Alagoas; conhecer a influência da idade (em anos completos) para o risco de desenvolvimento das lesões intraepiteliais de alto grau em mulheres com diagnóstico colpocitológico de atipias de significado indeterminado; e, conhecer a influência de agentes etiológicos para doenças sexualmente transmissíveis sobre o risco de desenvolvimento das lesões intraepiteliais de baixo e alto grau em mulheres com diagnóstico colpocitológico de atipias de significado indeterminado.

\section{MÉTODOS}

\section{Tipo de estudo}

Trata-se de um estudo transversal de prevalência onde se investiga a presença de lesões de alto grau em mulheres com atipias de significado indeterminado em 2007, no Município de Maceió, Alagoas. 


\section{Local do estudo}

Programa de Atenção à Saúde da Mulher, Posto de Atendimento Médico Salgadinho, situado na Rua Misael Domingos s/n, no bairro do Poço, cidade de MaceióAL; trata-se de uma unidade ambulatorial especializada de atendimento das ações e serviços de saúde, mantida pelo Sistema Único de Saúde - SUS e criada pelo governo federal em 1981. São atendidos todos os usuários do SUS da capital e do interior do Estado, sendo o segundo maior posto de atendimento médico do País e o primeiro em Alagoas, com o apoio da Prefeitura de Maceió, por meio da Secretaria Municipal de Saúde.

O PAM-Salgadinho, além de prestar assistência à saúde, tem o papel de prestador de serviços à população, funciona como um articulador entre as unidades básicas de saúde e as unidades de maior complexidade, nos casos de encaminhamentos e tratamentos em graus mais elevados, inclusive intermediando acomodação de pacientes em albergues, quando necessário.

Foi selecionado como local para o desenvolvimento deste trabalho por ser representativo do Estado de Alagoas e ser referência municipal e estadual para os casos de neoplasias cervicais.

\section{População e amostra do estudo}

A população do presente estudo constituiu-se de 253 prontuários de mulheres com diagnóstico colpocitológico de atipias de significado indeterminado que foram atendidas no PAM-Salgadinho, seguindo o protocolo do Ministério da Saúde, no período de um ano. Não há sistema informatizado no Bloco C do PAM-Salgadinho e, para a coleta de dados, os prontuários deveriam estar completos e disponíveis, por isso em razão da disponibilidade dos prontuários escolhemos o ano de 2007 para a pesquisa.

Todas as mulheres elegíveis (critério de inclusão) para o estudo realizaram o rastreamento colpocitológico, por meio de esfregaço corado pelo método de Papanicolaou, sendo o diagnóstico citológico fornecido pelo laboratório da Santa Casa de Misericórdia de Maceió. Os resultados da série de colpocitologias oncóticas, colposcopias e avaliações histológicas foram obtidos pela revisão dos prontuários das mulheres atendidas no referido Programa em 2007.

\section{Cálculo de tamanho da amostra}

Uma amostra sistemática foi utilizada, sendo utilizados todos os prontuários clínicos de mulheres portadoras de colpocitologia oncótica classificada, como atipia escamosa de significado indeterminado, atendidas no Programa de Atenção à Saúde da Mulher, Posto de Atendimento Médico Salgadinho, Maceió, Alagoas, no ano de 2007.

Variáveis selecionadas para o estudo

Idade em anos completos; tempo (em meses) entre a realização do exame Papanicolaou, a obtenção do resultado citológico, realização e resultado da biópsia cervical; alterações em células epiteliais escamosas: atipias de significado indeterminado; lesões escamosas intraepiteliais de baixo grau; lesões escamosas intraepiteliais de alto grau; carcinoma "in situ"; carcinoma escamoso invasor; agentes etiológicos de doenças sexualmente transmissíveis: (Human papiloma vírus; Gardnerella vaginalis; Tricomonas vaginalis); número de vezes que a mulher esteve grávida, independente da evolução da gestação.

\section{Coleta dos dados}

Os dados foram coletados pela autora do trabalho, após obtenção da autorização pelo serviço de saúde e aprovação do projeto de pesquisa pelos Comitês de Ética em Pesquisa da Universidade Federal de São Paulo e da Universidade de Ciências da Saúde de Alagoas.

\section{Técnica de coleta dos dados}

Os dados foram coletados de forma manual em um formulário padronizado com variáveis selecionadas para o estudo e armazenados em uma planinha eletrônica de dados (Microsoft Excelß 2003. Redmond, WA, EUA), nesse instrumento cada linha corresponde a um formulário de coleta de dados e cada linha aos dados coletados. Duas entradas de dados foram realizadas por diferentes digitadores, de forma independente e cega.

Os dados foram registrados em planilha eletrônica utilizando-se o programa Excel Microsoft Office 2000'TM.

\section{Análise estatística}

Para análise dos dados, foi apresentada a estatística descritiva. Para as variáveis qualitativas, foram apresentadas as frequências absolutas e relativas. Às variáveis quantitativas, foram utilizadas como medidasresumo a média, a mediana e o desvio-padrão, mínimo e máximo, para apontar a variabilidade.

Nas comparações, foi utilizado os testes Quiquadrado, ou Exato de Fisher (F), quando necessário. Para todos os testes considerou-se um nível de significância de $5 \%$. Desta forma, foram observadas às diferenças entre os grupos quando $\mathrm{p}$ valor foi menor que 0,05 (p-valor $<0,05$ ). Os resultados dos testes estão apresentados ao lado direito das tabelas.

\section{RESULTADOS}

Foram revisados 253 prontuários de mulheres com diagnóstico colpocitológico de atipias de significado indeterminado. A prevalência de lesões intraepiteliais foi de 60 casos (23,7\%), 14 com lesão de baixo grau, ou seja, neoplasia intraepitelial cervical grau I (NIC I) $(26,7 \%)$ e 46 com lesão de alto grau (NIC II e III) ou carcinoma (73,3\%), conforme os dados da Tabela 1. 
Tabela 1 - Mulheres, segundo tipo de lesão, atendidas no Programa de Atenção à Saúde da Mulher, PAM Salgadinho, Maceió - AL, 2007

\begin{tabular}{lrr}
\hline Tipo de lesão & $\mathbf{n}$ & $\mathbf{\%}$ \\
\hline Baix o grau & 14 & 26,7 \\
Alto grau e carcinoma & 46 & 73,3 \\
\hline Total & 60 & 100,0 \\
\hline
\end{tabular}

Neste estudo, o tempo médio para confirmação do resultado do diagnóstico de lesão foi de quatro meses. (Tabela 2).

Tabela 2 - Mulheres atendidas no Programa de Atenção à Saúde da Mulher segundo tempo (em meses) entre o resultado do exame citológico e realização da biópsia cervical e o resultado desta biópsia - PAM Salgadinho, Maceió - AL, 2007.

\begin{tabular}{|c|c|c|c|c|c|c|}
\hline Tempo & $\mathbf{n}$ & Média & $\begin{array}{l}\text { Medi - } \\
\text { ana }\end{array}$ & $\begin{array}{l}\text { Desvio- } \\
\text { padrão }\end{array}$ & Mín. & Max. \\
\hline $\begin{array}{l}\text { Entre o resultado } \\
\text { cito lógico e a } \\
\text { realiza ção d a } \\
\text { biópsia }\end{array}$ & 60 & 2,91 & 1,67 & 2,94 & 0,20 & 12,17 \\
\hline $\begin{array}{l}\text { Entre o resultado } \\
\text { cito lógico e o } \\
\text { resultado d a } \\
\text { biópsia }\end{array}$ & 60 & 4,42 & 3,53 & 3,15 & 0,63 & 13,83 \\
\hline
\end{tabular}

$\mathrm{Na}$ Tabela 3, observamos que a infecção pelo HPV parece estar associada a risco significativamente maior de lesões de alto grau e câncer invasivo comparado a única infecção.

Tabela 3 - Mulheres atendidas no Programa de Atenção à Saúde da Mulher, segundo variáveis qualitativas selecionadas - PAM Salgadinho, Maceió - AL,2007

\begin{tabular}{lrr}
\hline Variáveis & $\mathbf{n}$ & $\mathbf{\%}$ \\
\hline Prev alência do HPV & & \\
Não & 27 & 45 \\
Sim & 33 & 55 \\
Total & 60 & 100 \\
\hline Prevalência das lesões de baixo e alto grau & & \\
NIC I & 14 & 26,7 \\
NIC II & 24 & 40,0 \\
NIC III & 8 & 10,0 \\
Carcinoma in situ & 14 & 23,3 \\
Total & 60 & 100,0 \\
\hline Gardnerella vaginalis & & \\
Não & 58 & 96,7 \\
Sim & 2 & 3,3 \\
Total & 60 & 100,0 \\
\hline Tricomonas vaginalis & & \\
Não & 59 & 98,3 \\
Sim & 1 & 1,7 \\
Total & 60 & 100,0 \\
\hline
\end{tabular}

Todas as pacientes da amostra com resultado de ASCUS foram submetidas à colposcopia com realização de biopsia específica. A partir do resultado citológico, o tempo médio para realização da biópsia foi de três meses e o seu resultado com o laudo histopatológico quatro meses. (Tabela 4)

Tabela 4 -. Mulheres atendidas no Programa de Atenção à Saúde da Mulher, segundo as variáveis tempo (em meses) e o tipo de lesão - PAM Salgadinho, Maceió - AL, 2007

\begin{tabular}{lrrr}
\hline \multirow{4}{*}{ Tempo } & $\begin{array}{c}\text { Baixo } \\
\text { grau }\end{array}$ & $\begin{array}{c}\text { Alto grau e } \\
\text { carcinom a }\end{array}$ & $\begin{array}{c}\text { Valor } \\
\text { de } \boldsymbol{p}\end{array}$ \\
\cline { 2 - 3 } Entre o resultado citológico & & \\
e a realização da biópsia & & & \\
n & 14,0 & 46,0 & \\
Média & 2,8 & 2,9 & \\
Mediana & 1,4 & 1,8 & 0,924 \\
Desvio-padrão & 3,2 & 2,8 & \\
Mínimo & 0,2 & 0,2 & \\
Máximo & 12,2 & 10,6 & \\
Entre o resultado citológico & & \\
e o resultado da biópsia & & & \\
n & 14,0 & 46,0 & \\
Média & 4,5 & 4,3 & \\
Mediana & 3,7 & 3,1 & 0,776 \\
Desvio-padrão & 3,4 & 3,1 & \\
Mínimo & 0,7 & 0,6 & \\
Máximo & 13,8 & 11,5 & \\
\hline
\end{tabular}

Houve associação, estatisticamente significante na presença de HPV, evidenciando que há um maior percentual de mulheres com HPV entre as portadoras de lesão de baixo grau. Já nas infecções por Gardnerella $e$ Tricomonas vaginalis não foi observada significância. (Tabela 5)

Tabela 5 - Mulheres atendidas no Programa de Atenção à Saúde da Mulher, segundo a ocorrência de agentes etiológicos (HPV, Gardnerella e tricomonas vaginalis) e tipo de lesão. - PAM Salgadinho, Maceió - AL, 20072007

\begin{tabular}{|c|c|c|c|c|c|}
\hline \multirow{3}{*}{ Va riá veis } & \multicolumn{4}{|c|}{ Le sões } & \multirow{3}{*}{$\begin{array}{l}\text { Valor } \\
\text { de } p\end{array}$} \\
\hline & \multicolumn{2}{|c|}{$\begin{array}{c}\text { Baixo } \\
\text { grau }\end{array}$} & \multicolumn{2}{|c|}{$\begin{array}{l}\text { Alto grau e } \\
\text { carcinoma }\end{array}$} & \\
\hline & $\mathrm{n}$ & $\%$ & $\mathbf{n}$ & $\%$ & \\
\hline \multicolumn{6}{|l|}{$H P V$} \\
\hline Não & 1 & 18,8 & 26 & 53,5 & \multirow{2}{*}{0,017} \\
\hline Sim & 13 & 81,3 & 20 & 46,5 & \\
\hline \multicolumn{6}{|c|}{ Gardnella vaginalis } \\
\hline Não & 13 & 93,8 & 45 & 97,7 & \multirow{2}{*}{$0,472(\mathrm{~F})$} \\
\hline $\operatorname{Sim}$ & 1 & 6,3 & 1 & 2,3 & \\
\hline \multicolumn{6}{|c|}{ Tricomonas vaginalis } \\
\hline Não & 13 & 93,3 & 46 & 100,0 & \multirow[b]{2}{*}{-} \\
\hline Sim & 1 & 6,7 & - & - & \\
\hline
\end{tabular}

\section{DISCUSSÃO}

Em estudo realizado na cidade do Rio de Janeiro ${ }^{(7)}$, foram apreciados 127 casos de ASC-US e $57 \mathrm{com}$ diagnóstico de ASC-H. Foram considerados os 
diagnósticos histológicos ou, quando não cabível, o diagnóstico colposcópico. A prevalência encontrada de HSIL foi de 1,8\% nas citologias ASC-US e de 19,2\% nas citologias ASC-H . Não foram encontrados casos de câncer nesta amostra.

Neste trabalho, a prevalência de lesões intraepiteliais de baixo (LSIL) e alto graus (HSIL) foi elevada quando comparadas a outros estudos epidemiológicos sobre HSIL em mulheres com diagnóstico de células escamosas de significado indeterminado (ASCUS). A média de idade das pacientes do estudo foi de 37 anos, com intervalo de 18 a 75 anos, e a proporção das mulheres nas diferentes faixas etárias foi homogênea, o que poderia indicar a inexistência de predominância de uma faixa etária para alteração citológica ASCUS.

A incidência de lesões intraepiteliais em mulheres com idade inferior a 20 anos, as atipias em células escamosas de significado indeterminado foi encontrado por autores brasileiros em 5,5\% (22) da amostra, lesão intraepitelial escamosa de baixo grau em 28\% (113) e lesão intraepitelial escamosa de alto grau em $3 \%{ }^{(8)}$. Os mesmos autores consideram que a incidência de alterações citopatológicas é alta no início da vida sexual e associada à infecção pelo HPV.

Outros autores afirmam que o alto risco de infecção pelo HPV diminui com o aumento da idade em mulheres que apresentam células escamosas atípicas de significado indeterminado (ASCUS) ${ }^{(9)}$. Consideram ainda que, adicionando o teste de HPV no rastreamento histológico, a identificação de lesões de alto grau chega a $33 \%$ em comparação com a citologia isolada.

As mulheres que recebem um diagnóstico de HPV e ASCUS em países como os Estados Unidos da América, têm uma baixa prevalência. No entanto, têm definitivo risco para lesões de alto grau nos 24 meses que seguem com base em um diagnóstico anormal ${ }^{(10)}$. A idade não teve um impacto estatisticamente significativo sobre a probabilidade de diagnósticos da biópsia de NIC II e III e NIC I para estas pacientes.

Autores $^{(11)}$ determinaram a prevalência de HPV em 91\% dos casos e o HPV de alto risco foi detectado em todas as lesões intraepiteliais de alto grau (HSIL), 69\% de lesões intraepiteliais de baixo grau (SIL), 57\% de células escamosas atípicas de significado indeterminado (ASCUS) e $86 \%$ células escamosas atípicas, onde não é possível excluir uma lesão intraepitelial de alto grau (ASC-H).

A Sociedade Americana de Colposcopia e Patologia Cervical desenvolveu um algoritmo para gerenciar o exame citológico (Papanicolaou) ${ }^{(12)}$. Quando a amostra é insatisfatória, seja por falta de células endocervicais ou por inflamação, o exame deve ser repetido em seis meses. Se um teste de HPV foi realizado com o teste de Papanicolaou e voltou negativo, uma repetição do exame deve ser realizada a cada ano. Com um Papanicolaou insatisfatório, os clínicos devem tratar e repetir o exame em dois a quatro meses. Neste estudo, o tempo médio para confirmação do resultado do diagnóstico de lesão foi de quatro meses.

Em estudo realizado ${ }^{(13)}$, a prevalência do HPV em mulheres com diagnóstico de atipias de significado indeterminado ou de lesão intraepitelial de baixo grau foi de $69,9 \%$ e a média da idade 35,7. Os resultados sugerem que a infecção pelo HPV é múltipla e comum entre as pacientes submetidas à colposcopia por causa de ASCUS ou LSIL e parece estar associado com um risco significativamente maior de lesões de alto grau e câncer invasivo comparado à única infecção ${ }^{(13)}$, dados que corroboram com os resultados deste estudo.

O câncer cervical associado à gravidez é uma doença rara, com uma mediana de idade ao diagnóstico de 3035 anos e média de gestações $1,5^{(14-15)}$, dados que corroboram com nosso estudo.

Outros estudos relatam que a gravidez não afeta o resultado citológico materno ou biologia do tumor, na maioria desses trabalhos, foi avaliado um número pequeno de pacientes e não foram encontrados fatores de prognóstico em casos e controles ${ }^{(16-17)}$.

Autores ${ }^{(18)}$ compararam taxas de regressão às mulheres com HSIL pelo parto vaginal e cesáreo, foi encontrada regressão $67 \%$ e $13 \%$, respectivamente. No que diz respeito às lesões intraepiteliais, a reação inflamatória local relacionada secundariamente ao trauma cervical, durante o parto vaginal pode realmente melhorar a regressão da lesão ${ }^{(19)}$.

Em um estudo retrospectivo realizado com 40 mulheres foram analisadas as características do câncer cervical associado à gestação. Na população estudada, a idade média era de 33,5 anos e a média de gestações foi de 1,1. Constatou-se que o alargamento do colo uterino induzido pela gravidez pode diminuir relativamente a profundidade de invasão estromal em grávidas e que a metástase pode ocorrer no início da progressão da doença, mas, que pelo número limitado de pacientes, os resultados não podem fornecer diretrizes ao tratamento definitivo de mulheres com câncer cervical associado a gravidez ${ }^{(20)}$.

Observou-se que lesões neoplásicas são mais frequentes entre as terceira e quarta décadas de vida. Pelo número limitado de mulheres e as características inerentes a um desenho retrospectivo, nossos resultados demonstraram pouca influência no desenvolvimento da lesão e o número de gestações.

Muitas vezes, mulheres com carcinoma do colo do útero tinham uma flora vaginal de lactobacilos, normalmente, sem proteção. Neste contexto, a relação de inflamação bacteriana e (pré) neoplasia foram investigadas por autores ${ }^{(21)}$, que descobriram que as mulheres com uma citologia diagnosticada com desequilíbrio tiveram maior significância na (pré) neoplasia de colo do útero . 
Em contrapartida, autores indicaram que a vaginose bacteriana não mostrou nenhuma associação com a neoplasia intraepitelial cervical ${ }^{(22-23)}$. No presente estudo, a prevalência de Gardnerella e Tricomonas vaginalis foi, respectivamente, de $3,3 \%$ e $1,7 \%$, dados que corroboram com os achados na literatura.

Todas as pacientes da amostra com resultado de ASCUS foram submetidas à colposcopia com realização de biopsia específica. A partir do resultado citológico, o tempo médio para realização da biópsia foi de três meses e o seu resultado com o laudo histopatológico quatro meses.

Como a colposcopia pode ser um direcionador de condutas, tanto para as escamosas atípicas como às glandulares atípicas, a paciente que apresentar esta alteração citopatológica na Unidade Básica de Saúde deve ser encaminhada à Unidade de Referência de Média Complexidade para colposcopia imediata ${ }^{(24)}$.

O consenso americano de 2006 serviu de base para as recomendações brasileiras. São citadas as recomendações oriundas dos resultados do ALTS (ASCUS-LSIL Triage Study) ${ }^{(25)}$, um grande ensaio clínico randomizado patrocinado pelo National Institute of Health dos Estados Unidos da América (EUA) e que resultou em várias publicações.

O estudo ALTS demonstrou que a repetição da citologia, encaminhamento para colposcopia ou detecção de HPV oncogênico pela captura híbrida eram estratégias seguras e aceitáveis, quando o diagnóstico citológico inicial era ASCUS.

O custo dos testes de detecção de HPV nos EUA é muito inferior aos preços praticados no Brasil, além do alto custo dos exames colposcópicos no País. A repetição da citologia e o encaminhamento para colposcopia são considerados como aceitáveis e de menor custo no cenário brasileiro.

Para elaboração do Programa Nacional de Controle do Câncer do Colo do Útero (Viva Mulher), foram reunidos gestores, citopatologistas, colposcopistas e ginecologistas, representantes de associações médicas e

\section{REFERÊNCIAS}

1. Brasil. Ministério da Saúde. Instituto Nacional de Câncer. Estimativa 2010: incidência de câncer no Brasil [periódico na Internet]. Rio de Janeiro (RJ): Ministério da Saúde; 2009 [citado 2010 Fev 11]. Disponível em: http:// www.inca.gov.br

2. Brasil. Ministério da Saúde. Instituto Nacional de Câncer. Programa Nacional de Controle do Câncer do Colo do Útero e de Mama: Viva Mulher [Internet]. Rio de Janeiro (RJ): Ministério da Saúde; 2000 [citado 2010 Fev 11]. Disponível em: http://www.inca.gov.br

3. Brasil. Ministério da Saúde. Departamento de informática do SUS. Brasília (DF): Ministério da Saúde; 2009 [citado de serviços de alta e média complexidade que recebem mulheres com citologia alterada. Nas discussões, não foi abordada a viabilidade de incorporar novas tecnologias, como os testes de detecção de HPV oncogênico, questões de financiamento ou expectativas das mulheres. As recomendações americanas foram debatidas e adaptadas ao cenário brasileiro ${ }^{(26)}$.

Pode-se observar que houve associação estatisticamente significante na presença de HPV, evidenciando que há um maior percentual de mulheres com HPV entre as portadoras com lesão de baixo grau. Nas infecções por Gardnerella e Tricomonas vaginalis, não foi observada significância. Os limites dos resultados deste estudo estão relacionados ao baixo estabelecimento de relações causais e prognósticos, características dos estudos transversais. Ainda assim, os resultados apresentam implicações para a prática de enfermagem nos programas de rastreio do câncer cervical que atendem à população. Devem identificar as oportunidades de melhoria e foco nos esforços de forma adequada, a fim de obter uma eficácia máxima.

Possíveis estratégias para melhoria poderiam incluir a expansão, rastreamento e intervenções de prevenção às mulheres mais jovens, incluindo vacinação contra $O$ HPV, exame de Papanicolaou e educação em saúde.

\section{CONCLUSÕES}

A análise dos resultados obtidos com a realização deste estudo possibilitou as seguintes conclusões:

A prevalência de lesões intraepiteliais de alto grau em mulheres com diagnóstico colpocitológico de atipias de significado indeterminado (ASCUS) foi de 23,7\%, sendo elevada quando comparada a outras cidades brasileiras.

O aumento do risco de desenvolvimento de lesões de intraepiteliais de alto grau do colo uterino foi diretamente proporcional ao aumento da idade.

Maior prevalência de lesão intraepitelial de baixo grau associada à infecção pelo Papilomavirus humano.
2010 Fev 11]. Disponível em: http:/ / tabnet.datasus.gov.br 4. The 1988 Bethesda System for Reporting Cervical/ Vaginal Cytological Diagnosis. National Cancer Institute Workshop. Jama. 1989; 262(7):931-34.

5. Lambert B, Boivin Y, Lepage Y. Atypical Squamous Cells of Undetermined Significance (Ascus): Clinical cytological Significance. Int J Gynecol Cancer. 1999; 9(4): 329-32.

6. Brasil. Ministério da Saúde. Instituto Nacional Do Câncer. Nomenclatura Brasileira Para Laudos Citopatológicos Cervicais E Condutas Clínicas Preconizadas [periódico na Internet]. 2002 [citado 2010 Fev 11]. Rio de Janeiro (RJ): Ministério da Saúde. Disponível em: http:// http:// 
www.inca.gov.br

7. Cytryn A. Risco de lesão intra-epitelial de alto grau e câncer cervical nas pacientes com diagnóstico de células escamosas atípicas, quando não se pode excluir lesão intra-epitelial de alto grau [dissertação]. Rio de Janeiro: Fundação Oswaldo Cruz; 2008.

8. Monteiro DLM, Trajano AJB, Silva KS, Russomano FB. Incidence of cervical intraepithelial lesions in a population of adolescents treated in public health services in Rio de Janeiro, Brazil. Cad Saúde Pública [Internet]. 2009 [cited 2010 Jan 02]; 25(5): 1113-22. Available from: http:// www.scielosp.org/pdf/csp/v25n5/18.pdf

9. Silverloo I, Andrae B, Wilander E.Value of high-risk HPVDNA testing in the triage of ASCUS. Acta Obstetricia \& Gynecological Scandinavica [serial on the internet]. 2009 [cited 2010 Jan 01]; 88(9):1006-10. Available from: Academic Search Premier.

10. Armah H, Austin RM, Dabbs D, Chengquan Z. Followup Findings for Women With Human PapillomavirusPositive and Atypical Squamous Cells of Undetermined Significance Screening Test Results in a Large Women's Hospital Practice. Archives of Pathology \& Laboratory Medicine. 2009; 133(9):1426-30.

11. Said HM, Ahmed K, Burnett R, Allan BR, Williamson AL, Hoosen AA. HPV genotypes in women with squamous intraepithelial lesions and normal cervixes participating in a community-based microbicide study in Pretoria, South Africa. J Clin Virol. 2009; 44(4): 318-21.

12. American Society for Colposcopy and Cervical Pathology. Managing women with negative paps lacking endocervical cells or other quality indicators: pap adequacy algorithm. 2007 [cited 2009 Aug 06]; 11(4): 201-22. Available from : http://www.asccp.org/consensus/pap_adequacy/ pap_adequacy.pdf

13. Spinillo A, Dal Bello B, Gardella B, Roccio M, Dacco' MD, Silini EM. Multiple human papillomavirus infection and high grade cervical intraepithelial neoplasia among women with cytological diagnosis of atypical squamous cells of undetermined significance or low grade squamous intraepithelial lesions. Gynecol Oncol. 2009; 113(1):115-19.

14. Douvier S, Filipuzzi L, Sagot P. Management of cervical intra-epithelial neoplasm during pregnancy. Gynecol Obstet Fertil. 2003; 31:851-5.

15. Smith LH, Dalrymple JL, Leiserowitz GS, Danielsen B, Gilbert WM. Obstetrical deliveries associated with maternal malignancy in California. Am J Obstet Gynecol. 2001; 184:1504-12.
16. Clavel C, Masure M, Bory JP, et al. Human papillomavirus testing in primary screening for the detection of highgrade cervical lesions: a study of 7932 women. Br J Cancer. 2001; 84:1616-23.

17. Wright TCJ, Massad LS, Dunton CJ, et al. 2006 consensus guidelines for the management of women with abnormal cervical screening tests. J Low Genit Tract Dis. 2007; 11:201-22.

18. Siristatidis C, Vitoratos N, Michailidis E, et al. The role of the mode of delivery in the alteration of intrapartum pathological cervical cytological findings during the postpartum period. Eur J Gynaecol Oncol. 2002; 23:358-60.

19. Strinic T, Bukovic D, Karelovic D, Bojic L, Stipic I. The effect of delivery on regression of abnormal cervical cytological findings. Coll Antropol. 2002; 26:577-82.

20. Jong-Min Lee, Kwang-Beom Lee, Young-Tak Kim, HeeSug Ryu, Young-Tae Kim, Chi-Heum Cho, Sung-Eun Namkoong, Ki-Hun Lee, Ho-Sun Choi, Kyung-Tai Kim Mead PB. Cervical-vaginal flora of women with invasive cervical cancer. Obstet Gynecol. 2008; 52:601-4.

21. Verbruggen BSM, Boon ME, Boon LM. Dysbacteriosis and squamous (pre)neoplasia of immigrants and Dutch women as established in population-based cervical screening. Diagn Cytopath. 2006; 34:377-81.

22. Frega A, Stentella P, Spera G, Pace S, Cipriano L, Di Ruzza $\mathrm{D}$, et al. Cervical intraepithelial neoplasia and bacterial vaginosis: correlation or risk factor? Eur J Gynaecol Oncol. 1997; 18:76-7.

23. Boyle DC, Barton SE, Uthayakumar S, Hay PE, Pollock JW, Steer PJ, et al. Is bacterial vaginosis associated with cervical intraepithelial neoplasia? Int J Gynecol Cancer. 2003; 13:159-63.

24. Brasil. Ministério da Saúde. Secretaria de Atenção à Saúde. Instituto Nacional de Câncer. Coordenação de Prevenção e Vigilância. Nomenclatura brasileira para laudos cervicais e condutas preconizadas: recomendações para profissionais de saúde. Rev Bras Ginecol Obstet. 2006;28(8):486-504.

25. ASCUS-LSIL Triage Study (ALTS) Group. Results of a randomized trial on the management of cytology interpretations of atypical squamous cells of undetermined significance. Am J Obstet Gynecol. 2003; 188(6): 1383-92.

26. Russomano F, Monteiro ACS, Mousinho RO. O diagnóstico citológico de células escamosas atípicas: uma avaliação crítica das recomendações diagnósticas. Rev. Bras. Ginecol. Obstet. [serial on the Internet]. $2008 \mathrm{Nov}$ [cited 2010 Feb 06]; 30(11):573-82. Available from: http:// www.scielo.br/pdf/rbgo/v30n11/08.pdf 